\title{
Depression increases in patients with Parkinson's disease according to the increasing severity of the cognitive impairment
}

\author{
Em pacientes com doença de Parkinson, a depressão aumenta de acordo com o aumento
} da gravidade do comprometimento cognitivo

Marcos Hortes N. Chagas ${ }^{1}$, Tais S. Moriyama², André C. Felício ${ }^{3}$, Ana Luisa Sosa ${ }^{4}$, Rodrigo A. Bressan², Cleusa P. Ferri ${ }^{5}$

\begin{abstract}
Objective: To test the hypothesis that severity of cognitive impairment modifies the association between depression and Parkinson's disease (PD). Method: One-phase population-based door-to-door surveys. This is a secondary analysis of 1,451 people aged 65 years and older with cognitive impairment living in defined catchment areas. Depression was estimated according to ICD-10, self-reported PD, disability according to WHODAS-II and cognitive status according to the CSI-D. Results: The mean age of the sample was 79.3 years old and most (69\%) were women. Of the total sample, $16.1 \%$ had depression and it was significantly higher among participants with PD. There was an increase on the ORs of the association between depression and PD with decreased scores in the cognitive test (Adjusted OR from 0.98 to 8.04). Conclusion: The association between depression and PD increases with the severity of the cognitive impairment.
\end{abstract}

Keywords: parkinsonism, depression, cognitive impairment, Parkinson’s disease.

RESUMO

Objetivo: Testar a hipótese que a gravidade do prejuízo cognitivo modifica a associação entre depressão e doença de Parkinson (DP). Método: Estudo populacional através da análise secundária de 1.451 pessoas com idade maior ou igual a 65 anos com prejuízo cognitivo que residiam em áreas de abrangência definidas. A depressão foi estimada de acordo com a CID-10, auto-relato de DP, incapacidade conforme a WHODAS-II e nível cognitvo de acordo com a CSI-D. Resultado: A média de idade foi 79,3 anos, predominaram as mulheres (69\%). Do total de indivíduos, 16,1\% tinham depressão, significantemente maior entre os participantes com DP. Houve aumento gradativo na OR relativa à associação entre depressão e DP com a diminuição do escore no teste cognitivo (OR ajustado variou de 0,98 a 8,04). Conclusão: A associação entre depressão e DP parece aumentar com a gravidade do prejuízo cognitivo.

Palavras-chave: parkinsonismo, depressão, prejuízo cognitivo, doença de Parkinson.

Depression and dementia are common non-motor manifestations of Parkinson's disease (PD). Prevalence estimates vary widely, but most studies have suggested that $40 \%$ to $50 \%$ of $\mathrm{PD}$ patients present clinically relevant depression ${ }^{1,2}$ and $30 \%$ of them present dementia ${ }^{3}$.
Cognitive impairment itself increases the risk of depression $^{4}$ and thus it is plausible that the presence of cognitive impairment might influence the association of depression with PD. Most studies investigating the association of depression and PD are based on convenience clinical samples and

\footnotetext{
${ }^{1}$ Departamento de Neurociências e Ciências do Comportamento, Universidade de São Paulo, Ribeirao Preto SP, Brazil;

${ }^{2}$ Departamento de Psiquiatria, Laboratório Interdisciplinar de Neurociências Clínicas, Universidade Federal de São Paulo, Sao Paulo SP, Brazil;

${ }^{3}$ Instituto Israelita de Ensino e Pesquisa, Hospital Israelita Albert Einstein, Sao Paulo SP, Brazil;

${ }^{4}$ National Institute of Neurology and Neurosurgery of México, Mexico City, México;

${ }^{5}$ Departamento de Psicobiologia, Universidade Federal de São Paulo, Sao Paulo SP, Brazil.
}

Correspondence: Marcos Hortes N. Chagas; Hospital das Clínicas; Av. Bandeirantes, 3900 / $3^{\circ}$ andar; 14048-900 Ribeirão Preto SP, Brasil; E-mail: mchagas@ fmrp.usp.br

Conflict of interest: The 10/66 Dementia Research Group's research has been funded by the Wellcome Trust Health Consequences of Population Change Programme (GR066133 - Prevalence phase in Cuba and Brazil; GR08002- Incidence phase in Peru, Mexico, Argentina, Cuba, Dominican Republic, Venezuela and China), the World Health Organization (India, Dominican Republic and China), the US Alzheimer's Association (IIRG - 04 - 1286 - Peru, Mexico and Argentina), and FONACIT/ CDCH/ UCV (Venezuela). The Rockefeller Foundation supported the 10/66 dissemination meeting at their Bellagio Centre. Alzheimer's Disease International has provided support for networking and infrastructure. Tais S. Moriyama receives financial support from Instituto UNIEMP - Hospital Israelita Albert Einstein and is also supported by a doctorate scholarship from the CAPES. Rodrigo A. Bressan is an employee of the Federal University of São Paulo, receives financial support from Instituto UNIEMP - Hospital Israelita Albert Einstein and is recipient of a CNPq Research Productivity fellowship award.

Received 28 October 2013; Received in final form 28 February 2014; Accepted 20 March 2014. 
frequently exclude patients with dementia or cognitive impairment ${ }^{5,6}$. Although both cognitive impairment and depression are frequent comorbidities of PD, little is known on how the association of depression and PD varies according to cognitive impairment.

We estimated the association between depression and PD in a large community sample of 1,451 elderly with cognitive impairment and tested the hypothesis that the strength of the association between depression and PD increases by severity of cognitive impairment even after adjusting for disability, a potential mediator of this association.

\section{METHOD}

This study is a secondary analysis of the 10/66 Dementia Research Group population-based surveys. Further details on the protocol can be found elsewhere ${ }^{7}$.

Participants were recruited following informed signed consent. Studies were approved by local ethical committees as well as by the ethical committee at King's College London.

Setting and sample: All residents aged 65 years and older from 11 geographically defined catchment areas in India, China, Cuba, Dominican Republic, Venezuela, Mexico, and Peru were interviewed $(n=14,960)$ in one-phase populationbased cross-sectional door-to-door surveys. The analysis presented in this study is restricted to those with cognitive impairment $(n=1,451)$. Response rates were $80 \%$ and above in all sites. The same protocol was used in all countries allowing fair comparisons between sites.

\section{Measurements}

Parkinson's disease: participants and their informants were asked if a doctor had ever diagnosed the participant with PD. Possible answers were: No, probable or certain. Only those who answered "certainly yes" were considered as "self-reported PD".

Diagnosis of depression: In order to categorize depressed participants, International Classification of Diseases $10^{\text {th }}$ revision (ICD-10) criteria $^{8}$ were used. The ICD-10 divides depressive episodes into mild, moderate and severe, for this analyse we considered any current ICD-10 depressive episode, irrespectively of the severity. These were derived using a computerized algorithm applied to the geriatric mental state (GMS) ${ }^{9,10}$.

Cognitive impairment: the cognitive assessment was based on the "Community Screening Instrument for Dementia” (CSI-D), a 32 items cognitive test assessing naming, comprehension, memory, orientation, apraxia and constructional difficulty. It has shown very good psychometric properties for cross-cultural studies ${ }^{11,12}$. The CSI-D population normative data for Latin America, India and China for the 10/66 Dementia Research Group has been previously detailed described elsewhere ${ }^{13}$. Objective impairment was defined as a score 2 standard deviations (SDs) below the average obtained with the total sample $(n=14,960)$ and was used to select the sample included in this analysis. Dementia was determined according to the DSM-IV and/ or 10/66 dementia diagnosis ${ }^{14}$.

Disability: It was measured using the 12-item intervieweradministered World Health Organization Disability Assessment Schedule II (WHODAS-II) ${ }^{15}$, developed by World Health Organization as a culture-fair assessment to use in cross-cultural studies.

Sociodemographic status: Age of all participants was established from participant and informant report and an official ID document; when the information was not clear or there was a discrepancy, an event calendar was used. Level of education and gender were also recorded during the interview.

\section{Statistical analysis}

We described the participants' characteristics in participants with and without PD and estimated the association between depression and PD adjusted for socio-demographic characteristic and disability. Odds ratios (OR) are derived from logistic regression models with 95\% confidence intervals (95\% confidence interval (CI)). To explore how the association between depression and PD behaved according to levels of cognition we used the same model as before for each levels of cognitive impairment according to quartiles of scores on the CSI-D. In order to further understand how cognition affects the association between depression and Parkinson we repeat the same logistic regression models among people with and we have also conducted the same analysis among participants with and without dementia.

\section{RESULTS}

A total of 1,451 participants were classified as having cognitive impairment and were included in this analysis. The sample mean age was 79.3 (standard deviation (SD) 0.2) years old and most part of participants were women (69\%). Overall, educational levels were low and $61.1 \%$ did not complete primary school. Levels of disability were high, $38.3 \%$ of the whole sample and $54.8 \%$ of $\mathrm{PD}$ patients reported 15 or more days loss due to disability in the last month. Table 1 shows participants' characteristics according to the presence of PD. Groups with and without PD did not significantly differed regarding their mean age, gender, mean scores on cognitive tests and levels of education, but PD patients had much higher levels of disability. A total of $234(16.1 \%)$ individuals fulfilled criteria for depressive episode according to ICD-10 and 42 (2.9\%) reported having PD. 
Table 1. Demographics and clinical characteristics of participants according to PD status.

\begin{tabular}{|c|c|c|c|}
\hline & With PD & Without PD & Total \\
\hline & $\mathrm{N}=42$ & $N=1,409$ & $N=1,451$ \\
\hline Mean age (SD) & $78.5(1.15)$ & $79.4(0.21)$ & $79.3(0.20)$ \\
\hline Female N (\%) & $26(61.9)$ & 975 (69.2) & $1,001(69.0)$ \\
\hline \multicolumn{4}{|l|}{ Education N (\%) } \\
\hline None or incomplete primary & $18(42.9)$ & $855(61.6)$ & $873(61.1)$ \\
\hline Complete primary & $17(40.5)$ & $328(23.6)$ & $345(24.1)$ \\
\hline Complete secondary & $4(9.5)$ & $132(9.5)$ & $136(9.5)$ \\
\hline Complete tertiary & $3(7.1)$ & $73(5.3)$ & $76(5.3)$ \\
\hline CSI-D mean scores (SD) & $23.2(1.08)$ & $21.0(0.22)$ & $21.4(0.22)$ \\
\hline WHODAS-II mean scores (SD) & $54.0(4.14)$ & $38.0(0.81)$ & $38.5(0.80)$ \\
\hline 15 or more disability days N (\%) & $23(54.8)$ & $533(37.8)$ & $556(38.3)$ \\
\hline Depression N (\%) & $12(28.5)$ & $222(15.8)$ & $234(16.1)$ \\
\hline Dementia N (\%) & $24(57.1)$ & $928(64.8)$ & $952(64.6)$ \\
\hline
\end{tabular}

Depression was significantly more frequent among participants with PD, $28.5 \%$ (12) versus non-PD subjects $15.8 \%$ (222). Crude OR was 2.18 (95\%CI 1.10-4.32) and after adjusting for age, gender, disability and years of education the association of PD with depression remained significant (OR 2.19, 95\%CI 1.08-4.45).

Table 2 shows the association of PD and depression by quartiles of cognitive status (crude and adjusted for age, gender, level of education and functional disability). When divided by quartiles of cognitive status, PD was significantly associated with depression only among those most severely impaired (adjusted OR 8.04 95\%CI 1.57-41.15). There was a clear increase in the OR values with the decrease in cognitive test scores (from 8.04 among the most impaired to 0.98 to the least impaired). We have also looked this association among participants with and participants without dementia, which shows a much stronger association between depression and PD among people with dementia (adjusted $\mathrm{OR}=4.44$, 95\%CI 1.80-10.93) than among people with cognitive impairment but without dementia (adjusted OR=0.69, 95\%CI 0.18-2.55).

\section{DISCUSSION}

We studied the relationship between PD and depression in a community sample of 1,451 elderly subjects with cognitive impairment from seven different low and middleincome countries. We found a strong association between depression and PD, which was stronger among those with worse cognitive impairment and among those with dementia

In our sample around one third of those with PD were depressed. There was a strong positive association of PD and depression even after adjusting for age, gender, levels of education and disability. In a recent systematic review, Reijnders et al. (2008) $)^{2}$ found that estimates on the prevalence of depression in PD vary according to study methodology, being lower in community based compared to clinical samples. These authors found a mean prevalence of $8.1 \%$ for studies assessing major depression in PD patients from the general population. We used a structured interview in a community sample of cognitive impaired elderly and found a much higher prevalence (28.5\%), which could be explained by the fact that we had a cognitively impaired sample and we used ICD-10 for diagnosing depressive episode.

Small previous studies suggested that the association of depression and PD is stronger for those with cognitive impairment ${ }^{16,17,18}$, but none looked at this association according to the severity of the cognitive impairment in a community sample. When we divided our sample by quartiles of levels of cognitive impairment we found that the association of depression with PD increased with increased cognitive impairment. Cognitive impairment itself increases the risk for depression irrespectively of other

Table 2. Association of PD with depression according to levels of cognitive impairments and dementia status.

\begin{tabular}{|c|c|c|}
\hline \multirow{2}{*}{ Impairment (by Quartiles) } & (OR) & Multi-variable* adjusted OR \\
\hline & OR $(95 \% \mathrm{Cl})$ & OR $(95 \% \mathrm{Cl})$ \\
\hline Most impaired ( $1^{\text {st }}$ quartile) & $5.40(1.24-23.54)$ & $8.04(1.57-41.15)$ \\
\hline Intermediate Impaired ( $2^{\text {nd }}$ quartile) & $2.77(0.65-11.90)$ & $2.14(0.45-10.26)$ \\
\hline Less impaired ( $3^{\text {rd }}$ quartile) & $1.61(0.42-6.12)$ & $1.42(0.33-6.15)$ \\
\hline Least impaired ( $4^{\text {th }}$ quartile) & $1.32(0.36-4.87)$ & $0.98(0.24-4.02)$ \\
\hline With Dementia & $3.78(1.62-8.83)$ & $4.44(1.80-10.93)$ \\
\hline Without Dementia & $0.86(0.24-3.03)$ & $0.69(0.18-2.55)$ \\
\hline
\end{tabular}

OR: odds ratio; *adjusted by age, gender, level of education and functional disability. 
diseases ${ }^{4}$. It might be that either depression or cognitive decline are symptoms of a more severe presentation of $\mathrm{PD}$ or that the presence of depression leads to cognitive deterioration.

$\mathrm{PD}$ is the second most common neurodegenerative disorders after Alzheimer disease. Nevertheless overall, PD is a low prevalent condition and only few studies are based in community samples. One strength point of our study is that we used data from community-based surveys in seven countries. However, yet our sample included only 42 participants with PD, which brings some uncertainty to our estimates. Another limitation to be considered is that we used selfreport to identify participants with $\mathrm{PD}$, the same protocol was used in all sites. Also, diagnosis of PD is difficult to be ascertained and misdiagnosis is common even among specialists $^{19}$. Self-reported PD has been validated against post mortem neuropathological examination in a large prospective community based study ${ }^{20}$, showing that selfreported PD is a good predictor of PD. Moreover the prevalence of PD we found is very similar to PD prevalence among other populations of older adults.

The cognitive deficits found in PD depressed patients can be reversible with remission of depressive symptoms ${ }^{21}$, however large prospective studies are needed to test if treating depression in PD would reduce the likelihood of developing dementia. Our findings show that the strength of the association of depression with PD increases with the severity of cognitive impairment and is also stronger among people with dementia. It is difficult to disentangle how much of this association is due to the bad performance of people with depression in the cognitive tests, however it is still plausible to think that the prevalence of depression among people with PD might be underestimated if people with impaired cognition or dementia are excluded.

\section{References}

1. Nakabayashi TIK, Chagas MHN, Côrrea ACL, Tumas V, Loureiro SR, Crippa JAS. Prevalence of depression in Parkinson's disease. Rev Psiq Clin 2008;35:219-227.

2. Reijnders JS, Ehrt U, Weber WE, Aarsland D, Leentjens AF. A systematic review of prevalence studies of depression in Parkinson's disease. Mov Disord 2008;23:183-189.

3. Aarsland D, Beyer MK, Kurz MW. Dementia in Parkinson's disease. Curr Opin Neurol 2008;21:676-682.

4. Korczyn AD, Halperin I. Depression and dementia. J Neurol Sci 2009;283:139-142.

5. Chagas $\mathrm{MH}$, Crippa JA, Loureiro SR, et al. Validity of the $\mathrm{PHQ}-2$ for the screening of major depression in Parkinson's disease: two questions and one important answer. Aging Ment Health 2011;15:838-843.

6. Martinez-Martin P, Schapira AH, Stocchi F, et al. Prevalence of nonmotor symptoms in Parkinson's disease in an international setting; study using nonmotor symptoms questionnaire in 545 patients. Mov Disord 2007;22:1623-1629.

7. Prince M, Ferri CP, Acosta D, et al. The protocols for the $10 / 66$ dementia research group population-based research programme. BMC Public Health 2007;7:165.

8. Organisation WH. ICD-10 - Classifications of Mental and Behavioural Disorder: Clinical Descriptions and Diagnostic Guidelines. Geneva: World Health Organisation, 1992.

9. Copeland JR, Prince M, Wilson KC, Dewey ME, Payne J, Gurland B. The Geriatric Mental State Examination in the $21^{\text {st }}$ century. Int J Geriatr Psychiatry 2002;17:729-732.

10. Copeland JR, Dewey ME, Griffiths-Jones HM. A computerized psychiatric diagnostic system and case nomenclature for elderly subjects: GMS and AGECAT. Psychol Med 1986;16:89-99.

11. Hall KS, Gao S, Emsley CL, Ogunniyi AO, Morgan O, Hendrie HC. Community screening interview for dementia (CSI 'D'); performance in five disparate study sites. Int J Geriatr Psychiatry 2000;15:521-531.
12. Prince M, Acosta D, Chiu H, Scazufca M, Varghese M, Dementia Research G. Dementia diagnosis in developing countries: a crosscultural validation study. Lancet 2003;361:909-917.

13. Sosa AL, Albanese E, Prince M, et al. Population normative data for the 10/66 Dementia Research Group cognitive test battery from Latin America, India and China: a cross-sectional survey. BMC Neurol 2009;9:48.

14. Prince MJ, de Rodriguez JL, Noriega L, et al. The 10/66 Dementia Research Group's fully operationalised DSM-IV dementia computerized diagnostic algorithm, compared with the 10/66 dementia algorithm and a clinician diagnosis: a population validation study. BMC Public Health 2008;8:219.

15. Ustun TB, Saxena S, Rehm J, Bickenbach J. Are disability weights universal? WHO/NIH Joint Project CAR Study Group. Lancet 1999;354:1306.

16. Dissanayaka NN, O'Sullivan JD, Silburn PA, Mellick GD. Assessment methods and factors associated with depression in Parkinson's disease. J Neurol Sci 2011;310:208-210.

17. Holroyd S, Currie LJ, Wooten GF. Depression is associated with impairment of ADL, not motor function in Parkinson disease. Neurology 2005;64:2134-2135.

18. Tandberg E, Larsen JP, Aarsland D, Cummings JL. The occurrence of depression in Parkinson's disease. A community-based study. Arch Neurol 1996;53:175-179.

19. Hughes AJ, Daniel SE, Ben-Shlomo Y, Lees AJ. The accuracy of diagnosis of parkinsonian syndromes in a specialist movement disorder service. Brain 2002;125:861-870.

20. Foltynie T, Matthews FE, Ishihara L, Brayne C, Mrc C. The frequency and validity of self-reported diagnosis of Parkinson's Disease in the UK elderly: MR CFAS cohort. BMC Neurol 2006;6:29.

21. Boggio PS, Fregni F, Bermpohl F, et al. Effect of repetitive TMS and fluoxetine on cognitive function in patients with Parkinson's disease and concurrent depression. Mov Disord 2005;20:1178-1184. 